\title{
The Significance and Challenge of Quantitative Hemodynamic Study in Moyamoya Disease
}

W e read with great interest the article by Dlamini et $\mathrm{al}^{1}$ regarding the use of blood oxygen level-dependent (BOLD) MR imaging to predict the ischemic risk in children with Moyamoya disease (MMD). The authors demonstrated that idiopathic Moyamoya disease and the presence of steal are independently associated with ischemic events. We really appreciate the interesting observation in their conclusion. Meanwhile, after reading this article, we would like to highlight 2 important questions that it raises.

First, it is notable that the negative BOLD cerebral-vascular reactivity (CVR) or the "vascular steal" phenomenon occurs in 93.8\% of pediatric MMD patients with ischemic events. This suggests that the appearance of vascular steal represents the failure of the intracranial vascular autoregulation in MMD. MMD is a chronic, progressive disease. With the progressive stenosis of the internal carotid artery and the decrease of intracranial blood supply, radiologic ischemia could occur. Because of the vascular autoregulation, the intracranial vessels dilate reflexively to maintain cerebral blood flow perfusion. Patients can present no clinical symptoms, called "asymptomatic ischemia." However, this persistent chronic vasodilation will lead to the decrease or disappearance of CVR. Under the stimulation of a vasodilator, such as $\mathrm{CO}_{2}$ or acetazolamide, the blood is redistributed from the area where vasodilation is most serious to the area where vasodilation is still possible, namely "steal phenomenon." Therefore, the occurrence of steal phenomenon indicates the loss of the automatic regulation function of intracranial vessels. Coincidently, in a recent study, ${ }^{3}$ when the authors assessed CVR using xenonenhanced CT with and without acetazolamide, 40\% (77/192) of the hemispheres showed steal phenomena and all of them were symptomatic.

Second, across the years, many quantitative techniques for cerebral blood flow and other hemodynamic parameters have been developed, such as PET, SPECT, xenon-enhanced CT, dynamic perfusion CT, MR imaging with dynamic susceptibility contrast and arterial spin-labeling, and Doppler ultrasonography. ${ }^{2}$ However, all of these technologies have special advantages and disadvantages. To evaluate the practicability of a new technology, one must consider factors like invasion, safety, radioactivity, patient compliance, cost, and so forth. In present study, the authors adopted a method relying on MR imaging to detect the blood oxygen level to evaluate the CVR of patients with MMD, showing a good predictability for both pre- and postoperative ischemic events. However, a larger sample size and more clinical trials are needed to detect the value of the clinical application.

\section{REFERENCES}

1. Dlamini N, Slim M, Kirkham F, et al. Predicting ischemic risk using blood oxygen level-dependent MRI in children with Moyamoya. AJNR Am J Neuroradiol 2020;41:160-66 CrossRef Medline

2. Lee M, Zaharchuk G, Guzman R, et al. Quantitative hemodynamic studies in Moyamoya disease: a review. Neurosurg Focus 2009;26:E5 CrossRef Medline

3. Teo M, Furtado S, Kaneko OF, et al. Validation and application for the Berlin grading system of Moyamoya disease in adult patients. Neurosurgery 2020;86:203-12 CrossRef Medline

(D). $\mathbf{Y u}$

Department of Neurosurgery Zhongnan Hospital of Wuhan University Wuhan, China

(1) Q. Du Department of Rheumatology Xiangya Hospital, Central South University Hunan, China

(D). Chen Department of Neurosurgery Zhongnan Hospital of Wuhan University Wuhan, China 